\title{
Enablers And Barriers To Utilisation Of Available Formal Support By Institutionalised Individuals With Alzheimer's Disease And Related Dementias In Rural South Western Uganda: A Qualitative Descriptive Study
}

VALLENCE NIYONZIMA ( $\nabla$ vniyonzima@must.ac.ug )

Mbarara University of Science and Technology https://orcid.org/0000-0002-0665-5863

Samuel Maling

Mbarara University of Science and Technology

Zeina Chemali

Harvard Medical School

Research article

Keywords: Alzheimer's disease, Dementia, Rural communities, Uganda

Posted Date: October 1st, 2019

DOI: https://doi.org/10.21203/rs.2.15396/v1

License: (c) (1) This work is licensed under a Creative Commons Attribution 4.0 International License.

Read Full License 


\section{Abstract}

Background: The world's population is ageing resulting in a larger number of people living with dementia. Prevalence of dementia for persons aged above 60 years is expected to increase. There is paucity of information regarding formal support available for institutionalised individuals with AD/ADRD, enablers and barriers to its utilisation particularly South Western Uganda. Therefore this study, filling this knowledge gap, sought to assess formal support available for individuals with AD/ADRD, enablers and barriers to its utilisation in rural communities in South Western Uganda

Methods: This study was conducted in two nongovernmental community based facilities for the elderly in South Western Uganda employing a qualitative cross sectional study design. Purposive sampling was used to recruit caretakers of individuals with Alzheimer's disease and related dementias. A total of 28 in depth interviews and 6 key informant interviews were conducted.

Results: Three arching themes emerged: 1) Formal support services available, 2) Enablers and 3) Barriers to the utilisation of the formal support services in institutionalised patients with AD/ADRD in rural communities in South Western Uganda.

Conclusions: These findings highlight the importance of formal care in the long-term care of individuals with AD/ADRD. There is a dire need to equip caretakers involved in the care of patients with AD/ADRD with the required skill set and include formal support services in the mainstream primary health care to make the services accessible.

\section{Background}

Owing to increasing life-expectancy and declining death rate, the world's population is aging (1). The increased longevity has resulted in larger number of people living with non-communicable diseases, including dementia. Prevalence of dementia for those aged 60 years and above is $5-7 \%$ and is expected to keep increasing $(2,3)$. Furthermore, it is reported that $58 \%$ of individuals with dementia currently live in low and middle income countries (LMIC). This number is expected to rise to $71 \%$ by $2050(4,5)$.

Uganda is a developing country with a population of about forty five million people of whom approximately $4.6 \%$ is over 60 years $(6,7)$. A study in Uganda found that $13.2 \%$ of all elderly patients above 60 years of age admitted on non-psychiatric wards had dementia (8). According to Ugandan traditions, the elderly live with their families, and therefore the number of established institutions that provide care to elderly patients with dementia is limited.

Dementia describes a continuum of psychological, cognitive and behavioural manifestations such as loss of memory, problems with reasoning and communication, and personality changes impairing a person's functional abilities in a number of domains including activities of daily living (ADLs) (5). Alzheimer's disease (AD) is a progressive degenerative disease of the central nervous system. Individuals 
who have the moderate and severe forms of the disease often require a higher level of care by trained personnel $(9,10)$

Most studies focussing on care of individuals with AD/ADRD and resource utilisation have been conducted in high resource settings $(3,8)$. The resources needed to care for a patient with Alzheimer's disease depends on the stage of the disease. For example in the mild- moderate stages of Alzheimer's disease, adult day care may be adequate while severe forms require a high level of care in nursing homes $(11,12)$. In addition, a large proportion of people with AD/ADRD have not been formally diagnosed in LMIC (13). Culturally, in Uganda, symptoms of AD/ADRD are considered part of the normal process of aging (14) and the belief among caregivers and other family members is that nothing can be done in the formal health care sector for persons with AD/ADRD (15); greatly affecting health seeking behaviours.

Dementia is one of the conditions that contribute to care dependency among elderly people(15). Individuals with AD/ADRD have a variety of needs as a result of this care dependency (16). Support for patients with $A D / A D R D$ including formal support become increasingly necessary as the condition progresses (13). However, studies have found that individuals with dementia and their caretakers underutilize formal care (17). In addition, there is paucity of information regarding formal support available for institutionalised individuals with $A D / A D R D$, enablers and barriers to its utilisation particularly South Western Uganda. Therefore this study, filling this knowledge gap, sought to assess formal support available for individuals with AD/ADRD, enablers and barriers to its utilisation in rural communities in South Western Uganda (SWU).

\section{Methods}

This study aimed at assessing formal support available for individuals with AD/ADRD, enablers and barriers to its utilisation in rural communities in South Western Uganda. The study utilized a qualitative descriptive study design.

Setting: This study was conducted in two nongovernmental community based facilities for the elderly in South Western Uganda: Reach One Touch One Ministries (ROTOM) and St Ignatius home for the elderly. South Western Uganda is comprised of a number of ethnic groups including the Banyankole, Bakiga and Bafumbira. The main economic activities in this region are peasant farming and cattle keeping. According to Ugandan traditions, the elderly are used to living with their families, and therefore the number of established institutions that provide care to elderly patients with dementia is limited

Reach One, Touch One Ministries [ROTOM] located in Muhanga, Rukiga district- is a non-denominational Christian ministry dedicated to meeting the spiritual, social, physical and psychological needs of the elderly and their dependents so they may live dignified, independent and hopeful lives. It operates mainly as an outpatient facility where patients with symptoms of dementia and other illnesses are managed and thereafter go back to their community. The facility also has an outreach programs to bring services closer to the people. 
St. Ignatius home; founded by Mbarara Arch Diocese, is a residential facility for the elderly nuns and other religious people serving Ibanda catholic parish and caters for not only the physical and social needs of elderly, but also their spiritual needs as well.

\section{Participants and sampling}

The study involved caregivers of patients aged 60 years and above and the service providers from the selected facilities. Purposive sampling was employed to recruit study participants in each of the rehabilitation centres. A total of 28 in depth interviews and 6 key informant interviews were conducted.

We included only caregivers who had lived with individuals with dementia aged 60 years and above for at least 6 months. An arbitrary period of 6 months was considered sufficient for the caregivers to have a clear understanding of the formal support services that the patients had received from these facilities. The study excluded caregivers of known HIV positive patients with dementia and those with dementia secondary to severe physical illness or recent history of head injury because they needed a different care package.

Pre-testing: A pre-test was conducted prior to the main study. This was a mini study conducted to ensure comprehension and accuracy of the interview guide. Any irregularities raised in the interview guide were adjusted based on the feedback. The semi-structured interview guide was pre-tested on three caretakers of individuals with Alzheimer's disease and two service providers at St. Paul's Family for the elderly located in Ruti, Mbarara district for clarity to increase credibility of the study. Gaps identified in the data collection tool were corrected before proceeding with the data collection process, for instance, emphasizing participant's barriers and enablers to utilization of formal support services.

Data collection procedure: Key informant interviews were conducted for the service providers in the facilities to get an in depth understanding of the services they provide to individuals with AD/ADRD and the barriers to utilisation of the formal support services.

Using the interview guide, the researcher and the research assistants (RAs) conducted the interviews in Runyankole Rukiga, the local language mainly spoken in South Western Uganda and an audio recorder was used to record the responses after seeking consent from participants to record their voices. Each interview lasted about 25-30 minutes. In order to facilitate participation and ensure participants' privacy and confidentiality, interviews were held in a private place where the subjects' responses could not be heard and only the interviewer and the RA were privy to the patients' stories

\section{Data analysis}

Data analysis was done concurrently with data collection using thematic content analysis based on emerging themes. It was done in three phases, pre-analysis, material exploration, and result treatment. In pre-analysis, the researcher transcribed all of the interviews immediately after completing them. Thereafter, thematic analysis of the data was done using the highlighting approach. The researcher identified and organized significant statements and commonalities into themes representing important 
aspects of the formal support available and barriers to its utilisation immediately after each interview. Emergent themes were documented.

Rigor of research

This was achieved by translation and back translation of the data collection tools, conducting a pilot study before the main study, use of purposive sampling to select information rich cases and use of direct quotes from the participants to enable readers to judge the final themes. The interviews took up to one hour to build a trusting relationship which allowed participants' to have enough confidence and trust in the researcher and enough time to describe formal support available, enablers and barriers to its utilisation. Furthermore,an interview guide was used to collect information related to the research question and was developed based on the literature and the local context. To ensure trustworthiness of the findings, a clear description of the study site, participants' characteristics, data collection methods and analysis processes was done.

\section{Approvals and Ethical considerations}

The research protocol was approved by the Mbarara University of Science and Technology Research Ethics Committee (MUST REC). We obtained administrative clearance from the study sites.Informed consent was obtained from those who met the inclusion criteria. Participants were assured that participation in the study was voluntary and that they were free to withdraw at any time they wish so. Confidentiality was achieved by assigning participants' codes, and providing privacy.

\section{Results}

Three arching themes emerged: 1. Formal support services available 2. Enablers and 3. Barriers to the utilisation of the formal support services in institutionalised patients with AD/ADRD in rural communities in South Western Uganda. Under each theme there several sub themes and they are described below:-

\section{Theme 1: Available formal support services}

Participants outlined a number of formal support services provided by community and faith based institutions that offer specialized services to the geriatric population in South Western Uganda. The formal service offered included nutrition support, medical services, physiotherapy and psychosocial support. Details of each of these services are provided below:

\section{Medical services}

Medical services across the two facilities were provided by qualified nurses, clinical officers and medical officers. Participants from the two facilities revealed that they always received treatment from the facilities. They received a wide variety of medications such as analgesics and antibiotics for the different conditions that their clients/patients presented with. Medications were always available as evidenced by their excepts below 
'... They give him medicines each time we go to the facility. Even when they come to the community, they bring different medicines for him... they even put medicines in his ears because he does not hear well...'

Key informants confirmed the fact that patient received medical attention from the facilities. Patients were routinely reviewed from the facilities and drugs prescribed for them. ROTOM has a community outreach program, through which patients who could not easily access the facilities received medical related services from their respective communities during outreaches.

'...we have medication for every one depending on the condition. We even have a doctor who comes every month to check on us and if we have a serious case he is called immediately. Sometimes the doctors find these patients in their homes during our outreach program and treat them...'

\section{Physiotherapy}

A key informant from one facility stated that they used to have physiotherapy services for their clients a year ago. The other two facilities never had physiotherapy services. Physiotherapy was very useful for the patients who are unable to move on their own and those who use wheel chairs. However, the facility no longer provides physiotherapy services because the service provider left and no one else has the skills needed to deliver physiotherapy.

'...Regarding physiotherapy, there was a white lady who was training the nuns, she had materials for lifting, creams for massage and they had plays (drama) which they used to do and exercises. We no longer do physiotherapy for our patients because the white lady left and none of us has physiotherapy skills...'

\section{Nutrition support services}

The key informants revealed that they provide nutrition support services to the clients that come to their facilities. These services were provided by health care workers and ranged from providing information to the patients and caregivers about benefits of a balanced diet in some facilities to the actual preparation and serving of food to patients in other facilities. In addition, some facilities provided food stuffs such as maize flour, sugar, among others to the patients and their families.

'... First of all they get nutrition services like breakfast, lunch and supper. We prepare for them all the meals and we make sure we give them a balanced diet including fruits, vegetables and others...'

... We are given food stuffs like maize floor, beans, and others to prepare from home. They used to give us prepared food at the facility, but nowadays they no longer provide it to patients and their caregivers. These days they teach you how to prepare food and which food to prepare for the patients...'

Psychosocial support

Participants reported that they and the patients received psychosocial support services from the facilities such as counselling and prayer. This kind of support was provided by trained counsellors and spiritual 
leaders and included formal counselling and spiritual support. Only one facility however had a qualified counsellor who was providing formal counselling services.

'... Whenever he would refuse to take treatment, people (Health workers and spiritual leader from ROTOM) would come and talk to him and he accepts to take treatment...'

'... We have a counsellor who has been here for those who can talk to her but we also have priests who come to visit our patients for spiritual encouragement and prayer when things are not going on well but there is a lot to do for them...'

\section{Theme 2: Enablers to utilisation of formal support services}

Participants outlined a number of enablers for utilisation of the formal support services provided by community and faith based institutions that offered specialized services to the geriatric population in South Western Uganda. We grouped enabling factors as patient related, caregiver related and institutional related enablers. Details of each of these factors are provided below

\section{Patient related enablers}

Participants reported that a number of factors enabled their patients to use the formal support services in the different facilities. These factors included knowledge of the existence of the services, perceived need for the services, perceived usefulness of the services and the belief that these services would improve quality of life for the patients. Most of the participants reported that when patients' quality of life and mood improved, they were encouraged to continue using the services.

'... She was treated from somewhere and her condition failed to improve so they referred us to this facility where she got better... Patient always wants to go there; the services have greatly improved his quality of life...'

Participants reported that the services their patients received helped to improve the health status of the patients and their quality of life. They further reported that the services are readily available and free of charge.

... The services my patient receives are important... very important because we can't afford, you find you don't have any single coin and they help us get transport and drugs, it's very important...

Caregiver related enablers

Participants reported that patients are able to utilise the formal support services because of a strong family social support system. The family members support the care of the patient and mobilise resources needed for continuity of the care as shown in the excerpts below

'...The family members have been helpful... they mobilise resources needed to care for our mother...' 
Furthermore, caregivers find it easy to take patients to the different facilities, because the supplies are always available, health workers are available and willing to attend to the patients. This greatly reduces the waiting time.

'...When you reach the facility, health workers immediately take care of the patient... you do not spend a lot of time waiting for them...'

Institutional enablers

Participants identified institutional related enablers that allow their patients to freely utilise the formal support services provided in these facilities. These ranged from the availability of free services, creation of awareness in the community about the availability of services and conducting outreaches and home visits to bring the services closer to the people.

'... The services are given to us free of charge... You only need transport to take the patient to the facility. Sometimes they (Health workers) give you transport back home...'

'...The whole community around Muhanga is aware of the services ROTOM provides so it is easy for you to take your patient there when he/she falls sick...'

\section{Theme 3: Barriers to utilisation of formal support services}

This theme describes the barriers to utilisation of formal support services in rural communities in South Western Uganda. The theme emerged from 3 thematic categories and these are patient related barriers, care giver related barriers and institutional barriers

\section{Patient related barriers}

Participants reported a number of patient related barriers that influence utilisation of formal support services. Sometimes, the patients refused the services while other times, the patients will want a specific person to provide the service. If that specific person is not around, then they prefer to cancel or miss the service.

.... There are like two or three sister cannot allow those without veils to touch them that is also a challenge and we don't wish it to be like that they are our mothers... I saw one she refused food whenever you would go there to bath or dress her she would refuse and close the door and later alone they took the key and you would find her in the corridor or compound completely naked...'

Participants further reported that patients misplace their belongings and accuse caregivers of stealing them. This makes the caring role tiresome and difficult.

...Taking care of my grandmother is very hard...you can't do anything for her and she appreciates. When something gets misplaced she will say it's me who has stolen it and yet she forgot where she placed it... 
A number of caregiver related barriers were identified. Participants reported caregiver burden. They mentioned that the caregiver role is very demanding and does not allow them to do any other activity. This is demonstrated by the quote below

'...First of all I get tired I wake up at 5am and the way you have looked at them they all have to bath, dress and then clean the whole house go for mass... This is a lot of work and we are very few here...'

Some participants reported that memory loss is part of the normal process of aging and would not take their elderly persons to seek care since it is considered normal. This is derived from the community's perception.

'... The patient is very old and the community thinks this is part of the normal process of aging and therefore no need of seeking care...'

Furthermore, participants reported lack of social support from the rest of the family members. The role of caring for the elderly patient with dementia is normally left with a few family members which is challenging

..It is a bit challenging for me... the other family members are not directly involved in her care... the work is too much for me...

\section{Institutional barriers}

Participants identified a number of institutional related barriers to utilisation of formal support services. Access to facilities and financial resources were top barriers. Most of the participants noted that the facilities are far and getting there with appropriate transport is a huge challenge. At times, the family does not have the money to cater for transport. Having said that, it could also be particularly challenging to get transport even when the money is available to pay for the trip.

... The facility is a bit far from home so I have to get a car or boda boda (motorised taxi) to take her if there is any problem and that requires money for transport which may not be available.... Transport is a big problem in case the patient falls sick in the night like 3am it's not easy to get someone to take you to the facility even when you have the money...

\section{Discussion}

This study assessed the formal support available for individuals with AD/ADRD, enablers and barriers to its utilisation in rural communities in South Western Uganda (SWU). Medical services, nutrition support services, psychosocial support and physiotherapy services were identified as the available formal support services. The enablers and barriers were classified as patient related, caregiver related and institutional factors. 
This study identified medical services, nutrition support services, psychosocial support and physiotherapy service as the formal support services that were readily available for patients in the three community and faith based facilities that care for the geriatric population with dementia in south western Uganda. This is similar to the findings of Levesque, Harris (18) who reported availability of counselling services, nutrition services and medical services for the patients with dementia and their caregivers in a tertiary hospital in Iran.

A number of enablers and barriers that influence utilisation of these services were identified. Because these facilities were faith based, respondents had a positive belief that these services were beneficial to their patients. This motivated them to always encourage the patients to use the services. In addition, respondents reported improvement in the clinical status of their patients when they used these services from the facilities. This is consistent with findings of Jett (19) who reported that some African-American participants had a notion that cognitive impairment was an 'act of God' or a supernatural intervention hence such patients resorted to God when they started experiencing signs of impaired cognition.

Some participants perceived memory loss as part of the normal process of aging and would not take their elderly persons to seek care since it is considered normal. This is the community perception about ageing for most people in south Western Uganda. This is in agreement with the findings of Adelman, Blanchard (20) who reported that carers and those without direct care giving experience recognised dementia symptoms, such as memory loss, disorientation and loss of functional abilities among the elderly persons but these were not interpreted as being part of an illness but rather were thought to be part of a normal ageing process. On the contrary however findings by La Fontaine, Ahuja (21) did not suggest dementia symptoms as part of the natural consequence of ageing. The difference in the two findings can be attributed to the participants involved in the studies. The former involved informal caregivers while the later involved formal caregivers for dementia. Attribution of cognitive decline to the normal process of aging has been cited as a major barrier for utilisation of formal support services by individuals with dementia (22).

Our study demonstrated that most of the patients with dementia had family support which enabled them to use the formal care services in the community faith based organisations. The study further established that physical family support was particularly provided by daughters-in-law and grandchildren. In the initial stages of the disease, the support required is related to housework or home maintenance, but later as functional disabilities increase and restrict the parent's everyday activities, she or he may need personal care. According to the African culture, it is the responsibility of the children and their grandchildren to take care of their ageing parents $(23,24)$. A few participants however reported lack of family support which makes it harder for patients to access and use these services from these facilities. According to Banerjee, Samsi (25) family carers are the most important source of dementia care among people who are less likely to access health or social services. The elder care has truly suffered with the changes in family dynamics, young couple leaving to urban settings or the increased number of women not only working but also working for more hours and years as well as the rising demand for a flexible labor force, all resulting in less free time, longer commuting and greater residential separation distances 
between family members (26). While more and more people are in need of care, the number of care-givers is not increasing at a comparable rate, hence negatively affecting the care of the elderly persons with $A D / A D R D$.

\section{Conclusion}

This study found that medical services, nutrition support services, psychosocial support and physiotherapy service as the formal support services available for patients in the two community and faith based facilities that care for the geriatric population with dementia in South western Uganda. A number of enablers and barriers influenced the utilisation of these formal support services. These results further highlight the importance of formal care in the long-term care of individuals with AD/ADRD.

There is a dire need to equip caretakers involved in the care of patients with AD/ADRD with the required skill set since the family members provide most of the care. As much as more research is needed in this field, the authors press for the immediate and urgent need to have the formal support services included in the minimum health care package for the elderly persons and made available at all primary health care levels to increase accessibility of these services to the general population. Of course, this goes without saying that the need is dire to fund such programs and train the qualified personnel to run them.

\section{List Of Abbreviations}

AD - Alzheimer's disease

ADLs - Activities of Daily Living

ADRD - Alzheimer's disease and Related Dementias

AIDS - Acquired Immunodeficiency Syndrome

HIV - Human Immune Virus

LMIC - Low and Middle Income countries

MUST - Mbarara University of Science and Technology

RA - Research assistants

REC - Research Ethics Committee

SWU - South Western Uganda

UDHS - Uganda Demographic Health Survey

WHO - World Health Organisation 


\section{Declarations}

- Ethics approval and consent

This study was approved by the Mbarara University of Science and Technology Research Ethics Committee (Number--) on $26^{\text {th }}$ November 2018. We obtained administrative clearance from the two study sites.Informed consent was obtained from those who met the inclusion criteria. Participants were assured that their participation in the study was voluntary and that they were free to withdraw at any time they wish so. Confidentiality was achieved by assigning participants' codes, and providing privacy.

- Availability of data and materials

The recordings and the transcripts used and/or analysed during the current study are available from the corresponding author on reasonable request

- Competing interests

The authors declare no competing interests

- Funding

Research reported in this publication was supported by the Fogarty International Center and the National Institute on Aging of the National Institutes of Health under Award Number D43TW010128. NIH did not in any way influence the design of the study, collection, analysis, and interpretation of data and in writing the manuscript. The content is solely the responsibility of the authors and does not necessarily represent the official views of the National Institutes of Health

- Authors contributions

VN participated in the design, data collection, and analysis, interpretation of data and writing of the manuscript

SM participated in the design of the study, data analysis and manuscript writing

ZC participated in the design, data analysis, writing the manuscript and proofreading the manuscript.

\section{References}

1.Brookmeyer R, Johnson E, Ziegler-Graham K, Arrighi HM. Forecasting the global burden of Alzheimer's disease. Alzheimer's \& dementia. 2007;3(3):186-91.

2.Mavrodaris A, Powell J, Thorogood M. Prevalences of dementia and cognitive impairment among older people in sub-Saharan Africa: a systematic review. Bulletin of the World Health Organization.

2013;91:773-83. 
3. Wimo A, Jönsson L, Bond J, Prince M, Winblad B, International AD. The worldwide economic impact of dementia 2010. Alzheimer's \& Dementia. 2013;9(1):1-11. e3.

4.Paddick S-M, Longdon AR, Kisoli A, Dotchin C, Gray WK, Dewhurst F, et al. Dementia prevalence estimates in sub-Saharan Africa: comparison of two diagnostic criteria. Global health action. 2013;6(1):19646.

5.WHO. Dementia: a public health priority: World Health Organization; 2012.

6.UBOS. The National Population and Housing Census 2014-SubCounty Report, Kampala, Uganda. 2016.

7.UDHS. Uganda AIDS indicator survey 2011. Uganda. Ministry of Health ICF International: ICF International. 2012.

8.Namuli JD. A Case of Alzheimer's Dementia in Uganda. Brain Degeneration and Dementia in SubSaharan Africa: Springer; 2015. p. 247-54.

9.Musisi S, Jacobson S. Brain Degeneration and Dementia in Sub-Saharan Africa: Springer; 2015.

10.Pink J, O'Brien J, Robinson L, Longson D. Dementia: assessment, management and support: summary of updated NICE guidance. bmj. 2018;361:k2438.

11.Dias A, Patel V. Closing the treatment gap for dementia in India. Indian journal of psychiatry. 2009;51(Suppl1):S93.

12.Paraïso MN, Guerchet M, Saizonou J, Cowppli-Bony P, Mouanga AM, Nubukpo P, et al. Prevalence of dementia among elderly people living in Cotonou, an urban area of Benin (West Africa). Neuroepidemiology. 2011;36(4):245-51.

13.Black BS, Johnston D, Rabins PV, Morrison A, Lyketsos C, Samus QM. Unmet needs of communityresiding persons with dementia and their informal caregivers: Findings from the maximizing independence at home study. Journal of the American Geriatrics Society. 2013;61(12):2087-95.

14.Emmatty LM, Bhatti RS, Mukalel MT. The experience of burden in India: A study of dementia caregivers. Dementia. 2006;5(2):223-32.

15.Connell CM, Roberts JS, McLaughlin SJ, Carpenter BD. Black and white adult family members' attitudes toward a dementia diagnosis. Journal of the American Geriatrics Society. 2009;57(9):1562-8.

16.McLaughlin T, Feldman H, Fillit H, Sano M, Schmitt F, Aisen P, et al. Dependence as a unifying construct in defining Alzheimer's disease severity. Alzheimer's \& Dementia. 2010;6(6):482-93.

17.McCusker J, Cole MG, Voyer P, Monette J, Champoux N, Ciampi A, et al. Use of nurse-observed symptoms of delirium in long-term care: effects on prevalence and outcomes of delirium. International 
Psychogeriatrics. 2011;23(4):602-8.

18.Levesque J-F, Harris MF, Russell G. Patient-centred access to health care: conceptualising access at the interface of health systems and populations. International journal for equity in health. 2013;12(1):18.

19.Jett KF. Mind-loss in the African American community: Dementia as a normal part of aging. Journal of Aging Studies. 2006;20(1):1-10.

20.Adelman S, Blanchard M, Livingston G. A systematic review of the prevalence and covariates of dementia or relative cognitive impairment in the older African-Caribbean population in Britain. International Journal of Geriatric Psychiatry: A journal of the psychiatry of late life and allied sciences. 2009;24(7):657-65.

21.La Fontaine J, Ahuja J, Bradbury NM, Phillips S, Oyebode JR. Understanding dementia amongst people in minority ethnic and cultural groups. Journal of Advanced Nursing. 2007;60(6):605-14.

22. Wils M, Lisaerde J, Verbakel J. 02. 11: Improving advance care planning in patients with dementia in a nursing home: defining facilitating factors and barriers. European Geriatric Medicine. 2014(5):S58.

23.Brodaty $\mathrm{H}$, Thomson $\mathrm{C}$, Thompson $\mathrm{C}$, Fine $\mathrm{M}$. Why caregivers of people with dementia and memory loss don't use services. International journal of geriatric psychiatry. 2005;20(6):537-46.

24.Egbert N, Dellmann-Jenkins M, Smith GC, Coeling H, JOHNSON RJ. The emotional needs of care recipients and the psychological well-being of informal caregivers: Implications for home care clinicians. Home Healthcare Now. 2008;26(1):50-7.

25.Banerjee S, Samsi K, Petrie CD, Alvir J, Treglia M, Schwam EM, et al. What do we know about quality of life in dementia? A review of the emerging evidence on the predictive and explanatory value of disease specific measures of health related quality of life in people with dementia. International Journal of Geriatric Psychiatry: A journal of the psychiatry of late life and allied sciences. 2009;24(1):15-24.

26.Schneider NF, Limmer R. Job mobility and living arrangements. Tracing mobilities: Towards a cosmopolitan perspective. 2008:119-39. 